\title{
Hyperuricemia is a Adverse Prognostic Factor for Colon Cancer Patients
}

Jiang Yan

Chuming Zhu

Department of General Surgery, Liyang People's Hospital Affiliated to Nantong University, Liyang, 213300, People's Republic of China
Correspondence: Chuming Zhu Department of General Surgery, Liyang People's Hospital Affiliated to Nantong University, No. 70 Jianshexi Road, Liyang, 213300, People's Republic of China Email zhuchuming20000@I26.com
Objective: Hyperuricemia is linked to the prognosis of a number of cancers; however, its association with colon cancer survival has not been fully elucidated. To investigate whether hyperuricemia affects the prognosis of colon cancer, we conducted a retrospective study.

Methods: The study included age- and sex-matched colon cancer patients, of whom 60 patients were diagnosed with hyperuricemia, and 120 patients did not have hyperuricemia. The overall survival (OS) and disease-free survival (DFS) of these patients were evaluated by Kaplan-Meier (K-M) analysis. The association between the survival of colon cancer patients and hyperuricemia was analyzed using the Cox regression method after adjusting for tumor stage and grade and vascular infiltration.

Results: The K-M survival analysis supported that patients with hyperuricemia had poor OS ( $P$ for the Log rank test $=0.0008$ ) and DFS. As demonstrated by the univariate analysis, the presence of hyperuricemia was correlated with decreased OS $\left(\mathrm{HR}_{\mathrm{OS}}=2.09, P=0.002\right)$. Tumor grade and tumor stage were also found to be independent predictors for the prognosis of colon cancer patients. In addition, poor OS among patients with hyperuricemia was also confirmed in the adjusted analysis $\left(\mathrm{HR}_{\mathrm{OS}}=1.94, P=0.005\right)$.

Conclusion: Hyperuricemia has an adverse effect on the prognosis and survival of patients with colon cancer. Further studies evaluating the cellular and molecular mechanisms are needed to validate the prognostic value of hyperuricemia in colon cancer.

Keywords: colon cancer, hyperuricemia, survival, prognosis, uric acid, biomarker

\section{Introduction}

Colon cancer poses a major threat to public health and is the leading cause of cancer-related death worldwide. ${ }^{1}$ Although there has been progress in colon cancer diagnosis, patients with colon cancer still have a low survival rate, especially patients at an advanced stage. ${ }^{2,3}$ The discovery of factors associated with cancer prognosis might help develop therapies with higher efficiency and improve the prognosis of colorectal cancer patients. Many studies have suggested that metabolism-related factors exert vital effects on the prognosis of colon cancer. Previous studies have shown that colorectal cancer patients with diabetes mellitus have a higher mortality risk and lower disease-free survival rate than those without diabetes mellitus. ${ }^{4}$ Xiao et al confirmed that Exo70 was an independent prognostic factor in colon cancer. ${ }^{5}$ And Ren et al found high expression of PLAC1 was related to poor prognosis of colon cancer patients. ${ }^{6}$ In the last decade, the correlation between hyperuricemia and the incidence and mortality in multiple cancers has drawn much attention. 
Uric acid, found in serum, is a molecule possessing antioxidant and pro-oxidant properties. ${ }^{7,8}$ Hyperuricemia is characterized by elevated levels of uric acid in the serum, which enhances the local or systemic inflammatory response. ${ }^{9}$ Abnormal metabolism of serum uric acid may cause cardiovascular disease, respiratory disorders, renal disease and metabolic syndrome. ${ }^{10,11}$ Interestingly, uric acid may prevent cancer due to its antioxidant properties regardless of its pro-inflammatory effect. ${ }^{10-12}$ Conversely, a high level of uric acid present in the serum is independently correlated with increased mortality in cancer. ${ }^{13}$ However, the effect of hyperuricemia on the survival rate of colorectal cancer patients remains unclear. Here, we investigated the impact of hyperuricemia on the prognosis of colon cancer patients.

\section{Materials and Methods Research Design}

Patients with histologically diagnosed colon cancer (from July 2002 to June 2014) at our hospital were recruited in this retrospective cohort study. A total of 180 colon cancer patients, including 60 patients with hyperuricemia and 120 patients without hyperuricemia matched by age, gender, tumor location, and treatment methods, and so on. The study was conducted following approval from the local human research ethics committee. Informed consent forms were signed by all participants. All patients have strictly followed the treatment protocol in our hospital, which is comparable between the two groups. Patients were excluded if they had a previous history of diabetes mellitus, coronary artery disease, cerebrovascular diseases, or treatment for hyperuricemia or if they had no information on the levels of uric acid or were not followed up. We recorded the age, sex and race of patients, the date and time of diagnosis, the stage, size and grade of tumors, vascular infiltration, and level of serum uric acid. TNM staging system was adopted in the diagnosis of colorectal cancer, which was divided into I, II, III and IV stages according to the depth of primary tumor invasion, regional lymph node metastasis and distant metastasis. According to the College of American Pathologists and World Health Organization, grade is assessed by calculating the percentage of gland forming in the tumor. Low-grade tumors have over $50 \%$ gland forming (well or moderately differentiated) and high-grade tumors have less than $50 \%$ gland forming (poorly differentiated). ${ }^{14}$ Vascular infiltration was defined as tumor within a round or ovoid vessel-like structure containing elastic tissue fibers arranged in an orderly concentric fashion including intramural and extramural blood vessel invasion. ${ }^{15}$

\section{Uric Acid Assay}

Prior to therapy, blood samples were collected by a single peripheral venous puncture. The level of serum uric acid was determined using the EDTA hydrazine method. Patients with serum uric acid levels greater than 420 $\mu \mathrm{mol} / \mathrm{L}$ (male) or $360 \mu \mathrm{mol} / \mathrm{L}$ (female) were diagnosed with hyperuricemia.

\section{Outcome Estimation}

We investigated the correlation of hyperuricemia with the overall survival (OS) and disease-free survival (DFS) of colon cancer patients. Patients included in this study were followed-up quarterly for three years and then half-yearly. All patients receives follow-up till to death or the end of follow-up or loss of visit. The most recent follow-up record in this study was performed on May 20, 2019.

\section{Statistical Analysis}

The Kaplan-Meier method and the Log rank test were used to study the correlation of hyperuricemia with the OS of colon cancer patients. The hazard ratio (HR) with 95\% confidence interval (CI) was determined, and Cox regression analysis was also performed. Covariates consisted of age ( $\geq 59$ vs $<59$ ), sex (male vs female), tumor grade (low vs high), TNM stage (III/IV vs I/II), and vascular infiltration (Yes vs No). STATA (version 12.0) was used for estimation.

\section{Results}

Table 1 summarizes the characteristics of all colon cancer patients. Patients with hyperuricemia had tumor stages and grades and vascular infiltration results that were similar to those in patients without hyperuricemia. However, colon cancer patients with hyperuricemia had significantly shorter OS and DFS than those without hyperuricemia, as revealed by the Kaplan-Meier analysis (Figures 1 and 2). In addition, the univariate analysis revealed that hyperuricemia was correlated with a decreased OS rate in colon cancer patients $\left(\mathrm{HR}_{\mathrm{OS}}=2.09,95 \%\right.$ CI 1.32-3.31; $P=0.002$ ) (Table 2). Furthermore, the multivariate analysis showed that hyperuricemia remained correlated with poor OS in colon cancer patients after adjustment for factors including age, sex, tumor stage and grade, and vascular infiltration $\left(\mathrm{HR}_{\mathrm{OS}}=1.94,95 \%\right.$ CI $1.22-3.09 ; P=0.005$ ) (Table 2 ). The sensitivity analysis 
Table I Characteristics of Colon Cancer Patients

\begin{tabular}{|c|c|c|c|}
\hline Factors & $\begin{array}{l}\text { Hyperuricemia } \\
(60)\end{array}$ & $\begin{array}{l}\text { Normouricemia } \\
\text { (I20) }\end{array}$ & $P$ \\
\hline Age, n (\%) & & & 0.670 \\
\hline$<59 y$ & $36(60.0 \%)$ & 68 (59.4\%) & \\
\hline$\geq 59 y$ & $24(40.0 \%)$ & 52 (40.6\%) & \\
\hline Sex, n (\%) & & & 0.600 \\
\hline Female & 25 (4I.7\%) & 55 (45.3\%) & \\
\hline Male & 35 (58.3\%) & 65 (54.7\%) & \\
\hline $\begin{array}{l}\text { TNM stage, } \\
\text { n (\%) }\end{array}$ & & & 0.768 \\
\hline I & II (I8.3\%) & $20(16.6 \%)$ & \\
\hline II & $10(16.7 \%)$ & $24(20.0 \%)$ & \\
\hline III & 29 (48.3\%) & $62(51.7 \%)$ & \\
\hline IV & $10(16.7 \%)$ & 14 (II.7\%) & \\
\hline $\begin{array}{l}\text { Tumor grade, } \\
\text { n (\%) }\end{array}$ & & & 0.483 \\
\hline High & $4 \mathrm{l}(68.3 \%)$ & 88 (73.3\%) & \\
\hline Low & 19 (31.7\%) & 32 (26.7\%) & \\
\hline $\begin{array}{l}\text { Vascular } \\
\text { infiltration, n (\%) }\end{array}$ & & & 0.764 \\
\hline Yes & 8 (I3.3\%) & 18 (I5.0\%) & \\
\hline No & $52(86.7 \%)$ & 102 (85.0\%) & \\
\hline
\end{tabular}

also strongly supported the findings mentioned above (data not shown), and the overall data remains stable and unaffected. In addition, colon cancer patients with low-grade tumors and tumors of advanced TNM stage had poor

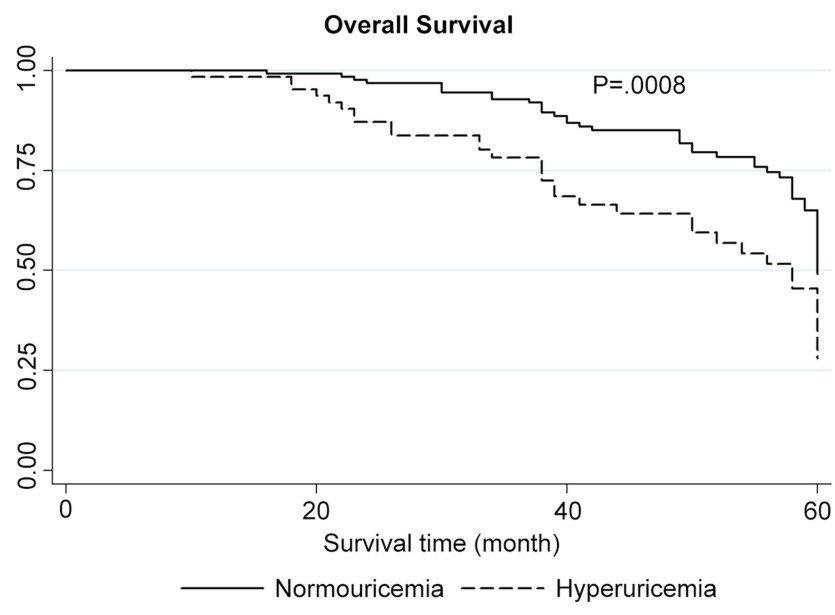

Figure I Kaplan-Meier overall survival (OS) analysis ( $P$ for the Log rank test equal to 0.0008$)$.

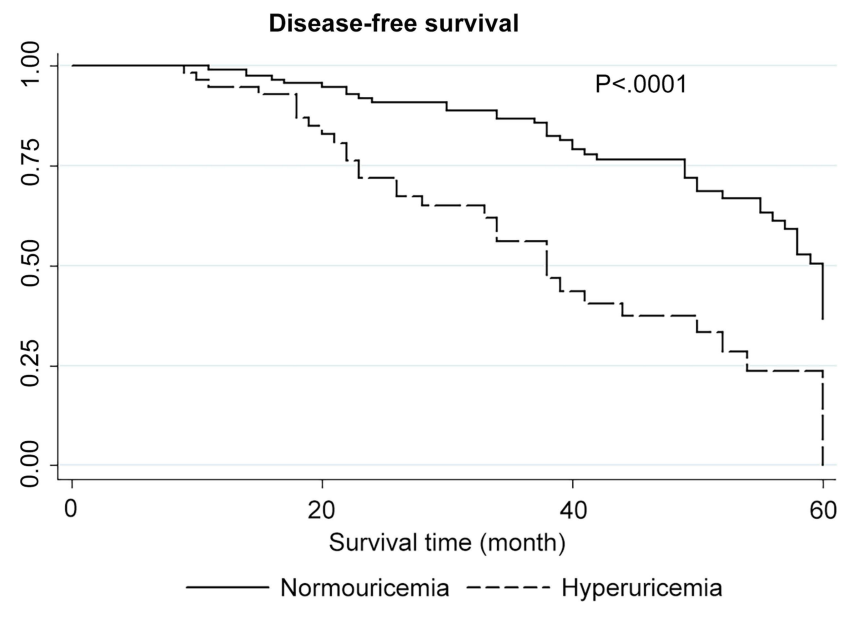

Figure 2 Disease-free survival (DFS) analysis.

prognosis (Table 2).Accordingly, hyperuricemia was independently associated with poor prognosis of colon cancer.

\section{Discussion}

Chemotherapy is the optimal treatment for colon cancer patients after surgery and those with advanced colon cancer. At present, researchers are still searching for prognostic biomarkers that can indicate the efficacy of chemotherapy for colon cancer patients. The development of optimized chemotherapy and personalized treatments for colon cancer will benefit from the identification of prognostic factors for tumor progression. ${ }^{16,17}$ In particular, currently available data reveal that metabolic disorders of serum uric acid promote the development and progression of malignant tumors. ${ }^{18,19}$ However, the role of serum uric acid in colon cancer prognosis remains largely unknown. In this retrospective cohort study, we investigated whether hyperuricemia affected the prognosis of colon cancer. Overall, patients suffering from both colon cancer and hyperuricemia had a poor prognosis compared to colon cancer patients without hyperuricemia, regardless of age, sex, TNM stage, tumor grade, and vascular infiltration.

Studies have shown that high levels of serum uric acid can promote inflammation, resulting in hyperuricemia, gout and cardiovascular complications. ${ }^{20-22}$ Increased proliferation of tumor cells may contribute to elevated levels of serum uric acid, leading to hyperuricemia, suggesting a potential link between tumors and uric acid metabolism. ${ }^{23}$ However, the role of serum uric acid in carcinogenesis remains obscure due to its antioxidant and pro-inflammatory properties. ${ }^{12}$ Owing to its antioxidant properties, serum uric acid can reduce oxidation-induced 
Table 2 Cox Regression Analysis

\begin{tabular}{|c|c|c|c|c|}
\hline & \multicolumn{2}{|c|}{ Univariate Analysis } & \multicolumn{2}{|c|}{ Multivariate Analysis } \\
\hline & HR $(95 \% \mathrm{CI})$ & $P$ & HR $(95 \% \mathrm{Cl})$ & $P$ \\
\hline Age ( $\geq 59$ years) & I.40 (0.89-2.20) & 0.149 & I.30 (0.82-2.07) & 0.268 \\
\hline Sex (male/female) & $1.14(0.72-1.80)$ & 0.582 & I.I3 (0.7I-I.80) & 0.597 \\
\hline Stage (III/IV) & $2.50(I .44-4.34)$ & 0.001 & $3.30(1.80-6.05)$ & $<0.001$ \\
\hline Vascular infiltration & $0.72(0.31-1.65)$ & 0.437 & I.02 (0.42-2.47) & 0.970 \\
\hline Tumor grade (low) & $1.36(0.85-2.17)$ & 0.202 & $2.07(1.25-3.44)$ & 0.005 \\
\hline Hyperuricemia (with/without) & $2.09(\mid .32-3.3 \mathrm{I})$ & 0.002 & $1.94(1.22-3.09)$ & 0.005 \\
\hline
\end{tabular}

Abbreviation: HR, hazard ratio.

cell damage. A number of studies have shown that hyperuricemia is also correlated with an increased risk of cancer. ${ }^{24,25}$ Furthermore, an increased level of serum uric acid is related to cancer outcomes, possibly reflecting more serious prognostic indications. ${ }^{24,25}$ Increased lysis of malignant cells can lead to hyperuricemia, which thus predisposes patients to an increased risk of renal failure and coronary heart disease. ${ }^{26,27}$ And Kalemkerian et al confirmed that renal insufficiency, elevated serum lactate dehydrogenase (LDH), and hyperuricemia were found in tumor lysis syndrome of solid tumors including colon cancer, which implied that tumor lysis syndrome is an important cause of hyperuricemia. ${ }^{28}$ Therefore, more data are needed to better interpret the underlying link between hyperuricemia and cancer.

Previous studies have suggested that hyperuricemia is correlated with the prognosis of several cancers. Tanriverdi et al found that non-small-cell lung cancer patients with hyperuricemia had a higher risk of tumor metastasis to the brain and poorer prognosis than patients without hyperuricemia. ${ }^{29}$ A recent study showed that hyperuricemia negatively impacts the prognosis of patients with osteosarcoma. ${ }^{30}$ However, increased levels of uric acid are correlated with improved clinical outcomes in patients diagnosed with soft-tissue sarcoma. ${ }^{31}$ We found that colon cancer patients with hyperuricemia had poor OS and DFS compared with those without hyperuricemia. Given that uric acid has diverse biological activities at different sites, such as serum, soft tissues and joints, an increase in uric acid from different origins might have varying effects on the development of tumors. Therefore, more studies are required to estimate the prognostic roles of uric acid in different malignant tumors.
The findings from this study must be interpreted cautiously due to the limitations of the cohort. The study was based on a retrospective design and employed a relatively small cohort to estimate cancer prognosis. In addition, different levels of serum uric acid might have different effects on the prognosis of patients with colon cancer. Future studies are encouraged to determine the precise prognostic role of different serum levels of uric acid in patients with colon cancer. Moreover, colon cancer patients with hyperuricemia receiving treatments and other comorbidities were excluded in this study, which might cause selection bias.

\section{Conclusion}

This study strongly suggests that hyperuricemia confers adverse effects on the prognosis of patients with colon cancer. Future studies are warranted to confirm the prognostic role of hyperuricemia, particularly regarding different levels of uric acid in the serum.

\section{Ethics Approval and Consent to Participate}

All patients in this research were informed of this study and signed consent forms. The study was approved by the ethics committee of Liyang People's Hospital. All studies were conducted in accordance with the Declaration of Helsinki. There were no unethical experiments performed in this study. And the ethical approval number is 2020042.

\section{Acknowledgments}

We are particularly grateful to all the people who have given us help on our article. 


\section{Funding}

There is no funding to report.

\section{Disclosure}

The authors declare that they have no competing interests.

\section{References}

1. Sideris M, Papagrigoriadis S. Molecular biomarkers and classification models in the evaluation of the prognosis of colorectal cancer. Anticancer Res. 2014;34(5):2061-2068. PMID: 24778007.

2. Cunningham D, Atkin W, Lenz HJ, et al. Colorectal cancer. Lancet. 2010;375(9719):1030-1047. doi:10.1016/S0140-6736(10)60353-4

3. Audisio RA, Papamichael D. Treatment of colorectal cancer in older patients. Nat Rev Gastroenterol Hepatol. 2012;9:716-725. doi:10.1038/nrgastro.2012.196

4. Mills KT, Bellows CF, Hoffman AE, Kelly TN, Gagliardi G. Diabetes mellitus and colorectal cancer prognosis: a meta-analysis. Dis Colon Rectum. 2013;56(11):1304-1319. doi:10.1097/ DCR.0b013e3182a479f9

5. Xiao L, Zheng K, Lv X, et al. Exo70 is an independent prognostic factor in colon cancer. Sci Rep. 2017;7(1):5039. doi:10.1038/s41598017-05308-X

6. Ren Y, Lv Y, Li T, Jiang Q. High expression of PLAC1 in colon cancer as a predictor of poor prognosis: a study based on TCGA data. Gene. 2020;763:145072. doi:10.1016/j.gene.2020.145072.

7. Glantzounis GK, Tsimoyiannis EC, Kappas AM, Galaris DA. Uric acid and oxidative stress. Curr Pharm Des. 2005;11(32):4145-4151. doi: $10.2174 / 138161205774913255$

8. Kang DH, Ha SK. Uric acid puzzle: dual role as anti-oxidantand pro-oxidant. Electrolyte Blood Press. 2014;12(1):1-6. doi:10.5049/ EBP.2014.12.1.1

9. Fang P, Li X, Luo JJ, Wang H, Yang XF. A double-edged sword: uric acid and neurological disorders. Brain Disord Ther. 2013;2(2):109. doi:10.4172/2168-975X.1000109

10. Colangelo LA, Gapstur SM, Gann PH, Dyer AR, Liu K. Colorectal cancer mortality and factors related to the insulin resistance syndrome. Cancer Epidemiol Biomarkers Prev. 2002;11 (4):385-391. PMID:11927499.

11. Horsfall LJ, Nazareth I, Petersen I. Serum uric acid and the risk of respiratory disease: a population-based cohort study. Thorax. 2014;69 (11):1021-1026. doi:10.1136/thoraxjnl-2014-205271

12. Ames BN, Cathcart R, Schwiers E, Hochstein P. Uric acid provides an antioxidant defense in humans against oxidant- and radical-caused aging and cancer: a hypothesis. Proc Natl Acad Sci U S A. 1981;78 (11):6858-6862. doi:10.1073/pnas.78.11.6858

13. Strasak AM, Rapp K, Hilbe W, et al. Serum uric acid and risk of cancer mortality in a large prospective male cohort. Cancer Causes Control. 2007;18(9):1021-1029. doi:10.1007/s10552-007-9043-3

14. Amri R, Bordeianou LG, Berger DL. Effect of high-grade disease on outcomes of surgically treated colon cancer. Ann Surg Oncol. 2016;23(4):1157-1163. doi:10.1245/s10434-015-4983-4

15. Minsky B, Mies C. The clinical significance of vascular invasion in colorectal cancer. Dis Colon Rectum. 1989;32(9):794-803. doi:10.1007/BF02562132

16. Chen J, Ye Y, Sun H, Shi G. Association between KRAS codon 13 mutations and clinical response to anti-EGFR treatment in patients with metastatic colorectal cancer: results from a meta-analysis. Cancer Chemother Pharmacol. 2013;71(1):265-272. doi:10.1007/ s00280-012-2005-9
17. Ye F, Liu Z, Tan A, Liao M, Mo Z, Yang X. XRCC1 and GSTP1 polymorphisms and prognosis of oxaliplatin-based chemotherapy in colorectal cancer: a meta-analysis. Cancer Chemother Pharmacol. 2013;71(3):733-740. doi:10.1007/s00280-012-2067-8

18. Yan S, Zhang P, Xu W, et al. Serum uric acid increases risk of cancer incidence and mortality: a systematic review and meta-analysis. Mediators Inflamm. 2015;2015:764250. doi:10.1155/2015/764250

19. Wang W, Xu D, Wang B, et al. Increased risk of cancer in relation to Gout: a review of three prospective Cohort Studies with 50,358 subjects. Mediators Inflamm. 2015;2015:680853. doi:10.1155/2015/ 680853

20. Okazaki H, Shirakabe A, Kobayashi N, et al. The prognostic impact of uric acid in patients with severely decompensated acute heart failure. $J \quad$ Cardiol. 2016;68(5):384-391. doi:10.1016/j. jjcc.2016.04.013

21. Li Q, Zhang Y, Ding D, et al. Association between serum uric acid and mortality among Chinese patients with coronary artery disease. Cardiology. 2016;134(3):347-356. doi:10.1159/000443518

22. Zhao G, Huang L, Song M, Song Y. Baseline serum uric acid level as a predictor of cardiovascular disease related mortality and all-cause mortality: a meta-analysis of prospective studies. Atherosclerosis. 2013;231(1):61-68. doi:10.1016/j.atherosclerosis.2013.08.023

23. Kuo CF, Luo SF, See LC, Chou IJ, Fang YF, Yu KH. Increased risk of cancer among gout patients: a nationwide population study. Joint Bone Spine. 2012;79(4):375-378. doi:10.1016/j.jbspin.2011.09.011

24. Strasak AM, Rapp K, Hilbe W, et al. The role of serum uric acid as an antioxidant protecting against cancer: prospective study in more than 28000 older Austrian women. Ann Oncol. 2007;18 (11):1893-1897. doi:10.1093/annonc/mdm338

25. Fini MA, Elias A, Johnson RJ, Wright RM. Contribution of uric acid to cancer risk, recurrence, and mortality. Clin Transl Med. 2012;1 (1):16. doi:10.1186/2001-1326-1-16

26. Baeksgaard L, Sørensen JB. Acute tumor lysis syndrome in solid tumors--a case report and review of the literature. Cancer Chemother Pharmacol. 2003;51(3):187-192. doi:10.1007/s00280-002-0556-x

27. Wannamethee SG. Serum uric acid and risk of coronary heart disease. Curr Pharm Des. 2005;11(32):4125-4132. doi:10.2174/ 138161205774913200

28. Kalemkerian GP, Darwish B, Varterasian ML. Tumor lysis syndrome in small cell carcinoma and other solid tumors. Am J Med. 1997;103 (5):363-367. doi:10.1016/s0002-9343(97)00153-8

29. Tanriverdi O, Cokmert S, Oktay E, et al. Prognostic significance of the baseline serum uric acid level in non-small cell lung cancer patients treated with first-line chemotherapy: a study of the Turkish Descriptive Oncological Researches Group. Med Oncol. 2014;31 (10):217. doi:10.1007/s12032-014-0217-z

30. Wang S, Liu X, He Z, Chen X, Li W. Hyperuricemia has an adverse impact on the prognosis of patients with osteosarcoma. Tumour Biol. 2016;37(1):1205-1210. doi:10.1007/s13277-015-3830-3

31. Szkandera J, Gerger A, Liegl-Atzwanger B, et al. Uric acid levels in blood are associated with clinical outcome in soft-tissue sarcoma patients. Clin Chem Lab Med. 2015;53(3):493-497. doi:10.1515/ cclm-2014-0486 


\section{Publish your work in this journal}

The International Journal of General Medicine is an international, peer-reviewed open-access journal that focuses on general and internal medicine, pathogenesis, epidemiology, diagnosis, monitoring and treatment protocols. The journal is characterized by the rapid reporting of reviews, original research and clinical studies

across all disease areas. The manuscript management system is completely online and includes a very quick and fair peer-review system, which is all easy to use. Visit http://www.dovepress.com/ testimonials.php to read real quotes from published authors.

Submit your manuscript here: https://www.dovepress.com/international-journal-of-general-medicine-journal 